Nouvelles perspectives en sciences sociales

\title{
Réalisme, sociologie et concepts de relations
}

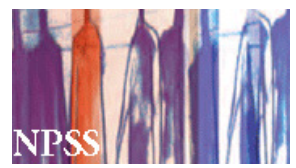

\section{Graham Scambler}

Volume 5, numéro 1, octobre 2009

Sur le thème de la relation

URI : https://id.erudit.org/iderudit/038624ar

DOI : https://doi.org/10.7202/038624ar

Aller au sommaire du numéro

\section{Éditeur(s)}

Prise de parole

ISSN

1712-8307 (imprimé)

1918-7475 (numérique)

Découvrir la revue

Citer cet article

Scambler, G. (2009). Réalisme, sociologie et concepts de relations. Nouvelles perspectives en sciences sociales, 5(1), 87-93. https://doi.org/10.7202/038624ar

\section{Résumé de l'article}

Le mot « relations " se réfère à de nombreux concepts qui sont parfois peu compatibles entre eux en sociologie contemporaine. Compte tenu de la brièveté de la contribution que nous offrons ici, aucun effort ne sera fait pour modifier cette situation. Je présenterai plutôt une interprétation générale, une sorte de dénominateur commun, puis j'illustrerai la manière dont j'ai cherché à utiliser ces termes dans mes propres travaux sur les stigmates et sur les inégalités en matière de santé au Royaume-Uni - dans mes écrits, les termes relations et structures sont plus ou moins traités en tant que synonymes. Je terminerai avec une réflexion sur ce que j'appelle le modèle jigsaw, un outil heuristique susceptible de faciliter l'étude sociologique des relations qui se manifestent dans le monde social que nous habitons. 


\title{
Réalisme, sociologie et concepts de relations
}

\author{
Graham Scambler \\ University College London (UCL)
}

$\coprod_{\text {parfois peu compatibles entre eux en sociologie contempo- }}$ raine. Compte tenu de la brièveté de la contribution que nous offrons ici, aucun effort ne sera fait pour modifier cette situation Je présenterai plutôt une interprétation générale, une sorte de dénominateur commun, puis j'illustrerai la manière dont j'ai cherché à utiliser ces termes dans mes propres travaux sur les stigmates et sur les inégalités en matière de santé au RoyaumeUni - dans mes écrits, les termes relations et structures sont plus ou moins traités en tant que synonymes. Je terminerai avec une réflexion sur ce que j'appelle le modèle jigsaw, un outil heuristique susceptible de faciliter l'étude sociologique des relations qui se manifestent dans le monde social que nous habitons.

L'insistance de Goffman à dire que le stigmate doit être compris, non pas en termes de défauts, d'attributs ou de traits physiques ahistoriques et transculturels, mais comme effets des relations sociales ${ }^{2}$, situe le point de départ de notre réflexion. Ce qui peut paraître comme intrinsèquement personnel ou indivi-

Traduit de l'anglais par Roger Gervais.

Erving Goffman, Stigma: The Management of Spoiled Identity, Harmondsworth, Penguin, 1968. 
duel est typiquement le produit du social. Ce constat sociologique fondamental a évidemment mené à une polémique entre les concepts du social ou des relations sociales. Mes propres approches partent d'un réalisme critique, une approche philosophique lancée par Bhaskar (1989) ${ }^{3}$. L'argument de base (kantien ou transcendantal) veut que les vrais objets, tant ceux des sciences de la nature que des sciences sociales sont, des mécanismes génératifs « réels » sous-jacents qui doivent exister si nous voulons avoir des chances d'expliquer la causalité observable des évènements via l'expérience, c'est-à-dire par les moyens empiriques dont nous disposons. De même, nous devons supposer que les relations stigmatisées doivent exister si nous voulons considérer et mieux comprendre les comportements des personnes (perçues comme) inacceptables.

Je suggère que les relations stigmatisées constituent une certaine part invariable de l'ensemble des relations sociales; il s'agit notamment des relations inégalitaires liées aux rapports de classes (émanant de l'économie) et d'autorité (émanant de l'État-nation). Ces relations stigmatisées opèrent aussi en lien avec les relations de déviance. Telles qu'elles sont présentées dans mes travaux, les premières se réferent à un "déficit ontologique ", à un "être " imparfait, alors que les relations de déviance se réferent à un "déficit moral » qui invoque une culpabilité . J'argumente que, dans le Royaume-Uni post-1970, une nouvelle dynamique entre les relations de classe et d'autorité mène à une revitalisation des relations de classes relatives à celles d'autorité et à un État qui est, paradoxalement, à la fois, plus orienté vers le marché libre et plus orienté vers la régulation et la surveillance. Cette nouvelle dynamique a précipité une transmutation du stigmate en déviance. En d'autres termes, ceux qui, souffrant de maladies chroniques ou de handicaps, avaient jusque-là accepté la stigmatisation, en mobilisant les normes de "la honte ", doivent de plus en plus assumer les accusations de la " classe de l'autorité " en

3 Voir Graham Scambler, "Sociology, social structure and health-related stigma ", Psychology, Health and Medicine, vol. 11, n 3, 2006, p. 288-295. Graham Scambler, "Health-related stigma ", Sociology of Health and Illness, vol. 31, no 3, avril 2009, p. 441-455. 
subissant les normes du "blâme ». Plus explicitement, l'État néolibéral ou l'État-nation discrimine et sanctionne certaines pratiques afin de réduire le coût de la main-d'œuvre et des transferts sociaux grâce à des "obligations de travail ", à la rénovation de politiques qui sont l'essence idéologique même du capitalisme. La manière de combattre ceux qui profitent de l'assistance sociale ou ceux qui la détournent est d'étendre les relations de déviance, politique dont la mise en application a été populaire. La " crise économique mondiale » actuelle, soulignant les indulgences coûteuses dont a bénéficié l'élite, pourrait ralentir ces tendances.

Cette même revitalisation profonde des relations de la classe de travail à la classe d'autorité est fondamentale pour comprendre l'évolution de mes théories sur les inégalités dans le domaine de

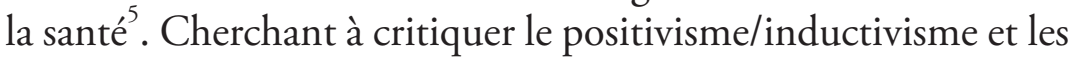
programmes de recherches de la socioépidémiologie orientés vers la prédiction, qui pourraient être considérés actuellement comme la «science normale », j'ai plaidé pour une approche sociologique explicite des relations sociales. En m'inspirant de la sociologie classique, j'avance que les découvertes provenant des épidémiologistes sociaux et de leurs alliés, qui montrent une relation statistique persistante entre les statut socioéconomiques et la santél longétivité, imposent une inférence rétroductive de l'existence de cette relation; elles imposent aussi un lien causal remarquable de la relation entre le groupe de classe (plutôt " catégorique " ou causalement efficace dans ses propres règles) et d'autorité (plutôt "dérivé » de la classe) pour les inégalités de santé dans le contexte ou de la configuration de l'État-nation occidental moderne. En bref, la relation entre classe et autorité doit exister et, à partir de cette asymétrie causale, si nous voulons analyser sociologiquement les inégalités de santé.

5 Graham Scambler, Health and Social Change: A Critical Theory, Buckingham, Open University Press, 2002; "Social structure and the production, reproduction and durability of health inequalities ", Social Theory and Health, vol. 5, no 4, novembre 2007, p. 297-315; "Capitalists, workers and health: Illness as a 'side-effect' of profit-making ", Social Theory and Health, vol. 7, n ${ }^{\circ}$, mai 2009, p. 117-128. 
La sociologie n'explique pas la totalité de l'histoire causale des inégalités sociales devant la santé. Non seulement la contingence et l'action (cette dernière étant toujours socialement structurée mais jamais structurellement déterminée) doivent être considérées, mais ce que nous caractériserons comme des mécanismes biologiques et psychologiques entrent aussi en jeu simultanément. Les mécanismes biologiques et psychologiques contribuent causalement au social de la même manière que les mécanismes sociaux contribuent causalement au psychologique et au biologique : en bref, causalité double, en amont et en aval ${ }^{6}$.

Le pouvoir explicatif de la sociologie dans le domaine des inégalités devant la santé est néanmoins considérable et peut être démontré. J'ai suggéré qu'en ce qui a trait aux inégalités devant la santé au Royaume-Uni, le processus de la nouvelle dynamique classe/autorité est fondé sur six types de "flux d'actifs " saillants pour la santé et la longévité :

1. Actifs biologiques (ou " corporels").

2. Actifs psychiques prédisposés à la résilience/vulnérabilité.

3. Actifs sociaux qui reflètent les niveaux d'intégration sociale, de réseautages et de soutiens.

4. Actifs culturels concernant les goûts et la facilité d'interaction.

5. Actifs spatiaux qui symbolisent les services locaux et la qualité de vie locale.

6. Actifs de biens matériaux qui se réferent au niveau de vie " relative ".

Comme les relations ou les structures sociales, les « flux d'actifs » ne sont pas facilement opérationnalisés. Non seulement ils vont varier dans le temps, mais ils sont rarement explicitement saisis, le degré ou la force du flux étant toujours un problème. De plus, ils interagissent. Ainsi, les "flux d'actifs » forts peuvent-ils com-

6 Graham Scambler, Panagiota Afentouli et Caroline Selai, «Discerning biological, psychological and social mechanisms in the impact of epilepsy on the individual: A framework and exploration ", dans Graham Scambler et Sasha Scambler (dir.), The Sociology of Chronic and Disabling Conditions: Assaults on the Lifeworld, London, Palgrave McMillan, à paraître.

7 Graham Scambler, "Health-related stigma ", op. cit. 
penser les plus faibles : un "flux d'actif » biologique réduit, par exemple, pourrait être compensé par un "flux d'actif » psychologique fort ou un arrêt du "flux d'actif » matériel, par des «flux d'actif " social ou culturel forts.

Si la nouvelle dynamique des relations entre les classes et les autorités a un pouvoir explicatif des inégalités en santé au Royaume-Uni et dans la plupart des États-nations, cela n'épuise pas la question. Il n'y a pas seulement des relations causales remarquables, ni des relations uniformes remarquables dans des contextes ou des configurations autres que les États-nations. Les relations de genre, d'ethnies et d'âges, par exemple, pourraient être des structures cruciales déterminant les flux d'actif qui contribuent au domaine de la santé dans certaines communautés, quartiers et foyers. C'est la nécessité d'éclairage empirique de telles variations et d'une telle complexité qui a conduit à développer le modèle jigsaw.

Le modèle jigsaw considère que les structures ou les relations sociales, par leur nature durable et comme de nombreux objets des sciences sociales ou de la nature, les aspects théoriques et donc nonobservables, possèdent une logique discernable au travers des effets de leurs relations sur des événements du monde. C'est la logique du régime de l'accumulation capitaliste, par exemple, qui produit les relations de classes pour lesquelles les pouvoirs causaux laissent des traces sur le quotidien. D'autres fortes logiques incluent le mode de régulation de l'État, le patriarcat, le tribalisme, la honte et le blâme, la soumission aux relations d'autorité, de genre, aux relations stigmatisées d'ethnicité et de déviance. Ces logiques et ces relations dyadiques doivent être étudiées à travers un ensemble de contextes sociaux ou de configurations sociales. Puisque les configurations, comme les sociétés, sont presque toutes des systèmes invariablement ouverts, il n'existe pas de dyades qui soient entièrement corrélées avec les événements qu'elles aident à expliquer.

Plus formellement, le modèle jigsaw comprend trois aspects :

(a) un modèle général du monde social contemporain;

(b) une série de modèles articulés en terme de logiques, 
de relations et de configurations, chacun constituant un morceau discret du jigsaw;

(c) un processus de raisonnement dialectique par lequel le modèle général informe l'application des modèles partiels et l'application des modèles partiels informe le portrait général.

L'un des avantages du modèle jigsaw est qu'il encourage une reconsidération des configurations à la lumière de multiples dyades de logiques et de relations. Il est évident, par exemple, que les inégalités de santé au Royaume-Uni ne peuvent ni être adéquatement expliquées de manière générale, pas plus que sociologiquement, en se référant uniquement à la logique du régime d'accumulation capital/mode de régulation et de ses relations classe/autorité, même si ces logiques et ces relations sont des causes de première importance. Afin d'en approfondir le pouvoir explicatif sociologique sur les inégalités dans la santé, cette même configuration doit être revisitée particulièrement en référence aux logiques/relations de patriarcat/genre et de tribalisme/ethnicité. En outre, bien que la logique/relation du régime d'accumulation du capital/classes et du mode de régulation/ autorité puisse venir en aide dans l'explication des inégalités dans le domaine de la santé dans la configuration des États-nations, elle pourrait être peu pertinente causalement pour les inégalités dans d'autres configurations, que ce soit dans une usine ou dans un foyer particulier. 


\section{Bibliographie}

Bhaskar, Roy, The Possibility of Naturalism (2nd Ed.), Hemel Hempstead, Harvester Wheatsheaf, 1989.

Goffman, Erving, Stigma: The Management of Spoiled Identity, Harmondsworth, Penguin, 1968.

Scambler, Graham, Health and Social Change: A Critical Theory, Buckingham, Open University Press, 2002.

Scambler, Graham, "Sociology, social structure and health-related stigma ", Psychology, Health and Medicine, vol. 11, n 3, 2006, p. 288-295.

Scambler, Graham, "Social structure and the production, reproduction and durability of health inequalities ", Social Theory and Health, vol. 5, no 4 , novembre 2007, p. 297-315.

Scambler, Graham, "Health-related stigma ", Sociology of Health and Illness, vol. 31, n 3, avril 2009, p. 441-455.

Scambler, Graham, "Capitalists, workers and health: Illness as a 'side-effect' of profit-making ", Social Theory and Health, vol. 7, n 2, mai 2009, p. 117-128.

Scambler, Graham, Panagiota Afentouli et Caroline Selai, "Discerning biological, psychological and social mechanisms in the impact of epilepsy on the individual: A framework and exploration ", dans Graham Scambler et Sasha Scambler (dir.), The Sociology of Chronic and Disabling Conditions: Assaults on the Lifeworld, London, Palgrave McMillan, à paraître. 